\title{
Cerebral haemodynamic effects in the human brain during radiation therapy for brain cancer
}

Myllylä, Teemu, Karthikeyan, Priya, Honka, Ulriika, Korhonen, Vesa, Karhula, Sakari, et al.

Teemu Myllylä, Priya Karthikeyan, Ulriika Honka, Vesa Korhonen, Sakari S. Karhula, Juha Nikkinen, "Cerebral haemodynamic effects in the human brain during radiation therapy for brain cancer," Proc. SPIE 11363, Tissue Optics and Photonics, 1136307 (2 April 2020); doi: 10.1117/12.2555892

SPIE. Event: SPIE Photonics Europe, 2020, Online Only, France 


\title{
Cerebral haemodynamic effects in the human brain during radiation therapy for brain cancer
}

\author{
Teemu Myllylä*a,b, Priya Karthikeyana, Ulriika Honka ${ }^{\mathrm{a}}$, Vesa Korhonen ${ }^{\mathrm{a}, \mathrm{c}, \mathrm{d}}$, \\ Sakari S. Karhula a,de, Juha Nikkinen ${ }^{\mathrm{a}, \mathrm{d}, \mathrm{e}}$ \\ ${ }^{a}$ University of Oulu, Research Unit of Medical Imaging, Physics and Technology, Oulu, Finland; \\ ${ }^{b}$ University of Oulu, Optoelectronics and Measurement Techniques Unit, Oulu, Finland; \\ 'Oulu University Hospital, Department of Diagnostic Radiology, Oulu, Finland; \\ ${ }^{\mathrm{d}}$ Medical Research Center (MRC), Oulu, Finland; \\ ${ }^{\text {e} O u l u}$ University Hospital, Department of Oncology and Radiotherapy, Oulu, Finland
}

\begin{abstract}
Radiotherapy is already well-established and an effective form of treatment for many cancers, especially for brain tumors. Currently, the clinical efficacy of a treatment, however, can only be established based on clinical or radiological responses observed after a significant period of time following the single irradiations during radiotherapy course. On the other hand, the radiotherapy dose is limited by its toxicity to surrounding healthy tissues. Particularly, radiation to brain tumors may sub-acutely or chronically affect cognition and cause fatigue even with conventional doses. However, there is currently no on-line and safe method to monitor the effects of radiation to the brain during the irradiation. In our project, we aim to develop an on-line method to monitor effects in brain tissue that correlate with the radiation dose in radiotherapy. In this case study, we use functional near-infrared spectroscopy (fNIRS) and study possible temporal effects in cerebral haemodynamics during irradiations of whole-brain radiotherapy (WBRT). fNIRS is safe for the patient, it can be used noninvasively and also in demanding environments, such as in radiotherapy treatment rooms during irradiation, and thus could be in future potential technique to be utilized for monitoring tailored radiotherapy.
\end{abstract}

Keywords: Radiotherapy, Irradiation, Haemoglobin, fNIRS, Brain

\section{INTRODUCTION}

fNIRS is commonly used in studies of cerebral blood oxygenation and tissue haemodynamic. In recent years, fNIRS has also started to attract attention as a potential tool for cancer diagnostics, particularly for breast cancer. Though clinical applications of fNIRS technology are still lacking, they may emerge in near future due to fNIRS' capability to detect parameters related to blood haemoglobin concentration. This is significant, because microvasculature and blood oxygenation concentrations of tumors differ from those of healthy tissue and such differences can be distinguished by fNIRS. So far, fNIRS has only been used to a limited extent in brain cancer research, but it may have potential for instance in preoperative localization of tumors [1] and in therapy monitoring. In comparison with fMRI, one of the main benefits of fNIRS is that it allows measuring concentration changes in both oxy-haemoglobin $(\mathrm{HbO})$ and deoxy-haemoglobin (HbR), while blood oxygenation level-dependent contrast functional fMRI (BOLD-fMRI) is only sensitive to changes in HbR concentration in blood. In addition, fNIRS system can be quite easily made compatible to the radiotherapy by using inside the treatment room only optical fibres designed for the purpose.

Cancer therapy treatments produce extensive changes in the physiological and morphological properties of tissues, including angiogenesis, hypoxia, alterations in cell nuclear size and density and denaturation [2]. Consequently, great clinical interest has been attached to measuring and tracking these biomarkers and changes. A key challenge involves developing tailored cancer therapy, geared toward considering individual biological responses during therapy. fNIRS offers an effective technique for monitoring such biomarkers, particularly tumor hypoxia and responses to chemo- and radiotherapy, which involves modulating tumor oxygenation to increase or decrease tumor hypoxia. This measurement is often performed by magnetic resonance imaging (MRI), which may not be an ideal solution. Howe et al. used fNIRS to determine absolute haemoglobin and changes in $\mathrm{HbR}$ and $\mathrm{HbO}$ in subcutaneous tumors in rodents. To better interpret MRI data, they paid particularly attention to factors that alter blood flow and oxygenation.

*teemu.myllyla@oulu.fi

Tissue Optics and Photonics, edited by Valery V. Tuchin, Walter C. P. M. Blondel,

Zeev Zalevsky, Proc. of SPIE Vol. 11363, 1136307 - (c) 2020 SPIE

CCC code: $0277-786 \mathrm{X} / 20 / \$ 21 \cdot$ doi: $10.1117 / 12.2555892$ 
Both carbogen and $\mathrm{O}_{2}$ breathing produced a significant reduction in $\mathrm{HbR}$ and an increase in $\mathrm{HbO}$, but a negligible change in total haemoglobin $(\mathrm{HbT})$. This contrasts with $\mathrm{N}_{2}$ breathing in terminal anoxia and intravenous hydralazine regime, which produced a negligible increase in $\mathrm{HbR}$, but large reductions in $\mathrm{HbO}$ and $\mathrm{HbT}$. Since $\mathrm{HbT}$ is proportional to blood volume, they suggested that large haemodynamic blood volume drops are likely to cause reduced arterial blood pressure. It then follows that MRI techniques that measure the R $2 *$ relaxation rate, which varies linearly with total $\mathrm{HbR}$, will underestimate the effects of hypotensive agents with increasing tumor hypoxia [3].

\subsection{Monitoring radiotherapy}

To the best of our knowledge there are no studies showing immediate haemodynamic effects during radiotherapy, particularly in human cancer therapy. This is probably due to the lack of appropriate technologies. Few studies have been conducted to investigate the haemodynamic and blood flow related parameters after the radiotherapy or between fractions (treatment sessions) but none during the actual irradiation.

A pilot study by Sunar et al. in 2006 investigated how patients with head and neck tumors responded to chemoradiotherapy. This approach provided different types of functional parameters i.e., blood flow, blood oxygenation, tumor oxygen metabolism, measured using diffuse reflectance spectroscopy (DCS) and fNIRS. The DCS setup included four receivers with a long coherence length laser operating at $785 \mathrm{~nm}$. The shortest and largest separation between source and detector fibers was 2 and $3 \mathrm{~cm}$, respectively. Also comprising four channels, the fNIRS instrument operated at the wavelengths of $690 \mathrm{~nm}, 785 \mathrm{~nm}$ and $830 \mathrm{~nm}$ using four source-detector distances $(1.8 \mathrm{~cm}, 2.2 \mathrm{~cm}, 2.6 \mathrm{~cm}$ and $3 \mathrm{~cm})$ to quantify oxygenation parameters. Significant changes in relative blood flow, tissue oxygen saturation and HbT were observed even in the first two weeks of treatment. The study protocol consisted of pre-radiation measurements providing baseline data and weekly follow-up optical measurements, conducted just before each new weekly treatment. Their preliminary results suggest diffuse optics-based therapy monitoring may have clinical promise [4].

Dong et al. used NIRS system combined with DCS to measure head and neck tumor blood flow and haemodynamic effects of chemoradiotherapy for the prediction of treatment outcome in a relatively large population $(\mathrm{n}=47)$. A commercial spatially resolved frequency domain based NIRS (Imagent, ISS, IL) included 16 laser diodes at 690, 750, 780, and $830 \mathrm{~nm}$ (four diodes for each wavelength) modulated at $110 \mathrm{MHz}$ as sources and one photomultiplier tube (PMT) as the detector. The measurements were performed prior to the treatment and weekly over the treatment period of 7 weeks. As a result, significant changes in tumor haemodynamics were found within the first 3 weeks of treatment, which shows promises that dynamic modification of tumor haemodynamics based on frequent optical measurements during chemoradiotherapy potentially can optimize treatment outcomes individually [5].

Nyström et al. used spectrometer, laser Doppler perfusion imaging and digital camera to study skin reaction, particularly for characterizing erythema, among patients undergoing radiotherapy treatment. The results from this preliminary study indicated that all three methods provide relevant information related to the radiation dose, in particular, the whole NIR spectra contained information associated with erythema [6].

In a recent paper by Mohammadkarim et al. a Doppler ultrasonography based analysis of common carotid arteries was studied when radiation was exposed to head and neck. Significant increases in the mean values of the Pulsatility Index and Resistive Index after radiotherapy were observed. They concluded that external radiotherapy involving the head and neck region may lead to carotid stenosis and failure of the blood supply in the short-term after cancer treatment. Further, the most interesting finding in this study was the identification of significant differences in haemodynamic parameters, especially blood volume flow, between baseline and post-treatment arteries [7].

\section{METHODOLOGY}

In WBRT treatment radiation dose is delivered externally using linear accelerators. During the treatment session, the patient is typically in a supine position while radiation fields are targeted in the brain, see Figure 1. In our study, all the measurements were performed with the linear accelerator (TrueBeam, Varian Medical Systems). Forward-Intensity Modulated Radiation (FIMRT) technique was used with 6 MV X-ray beams at the dose rate of $600 \mathrm{MU} / \mathrm{min}$. 

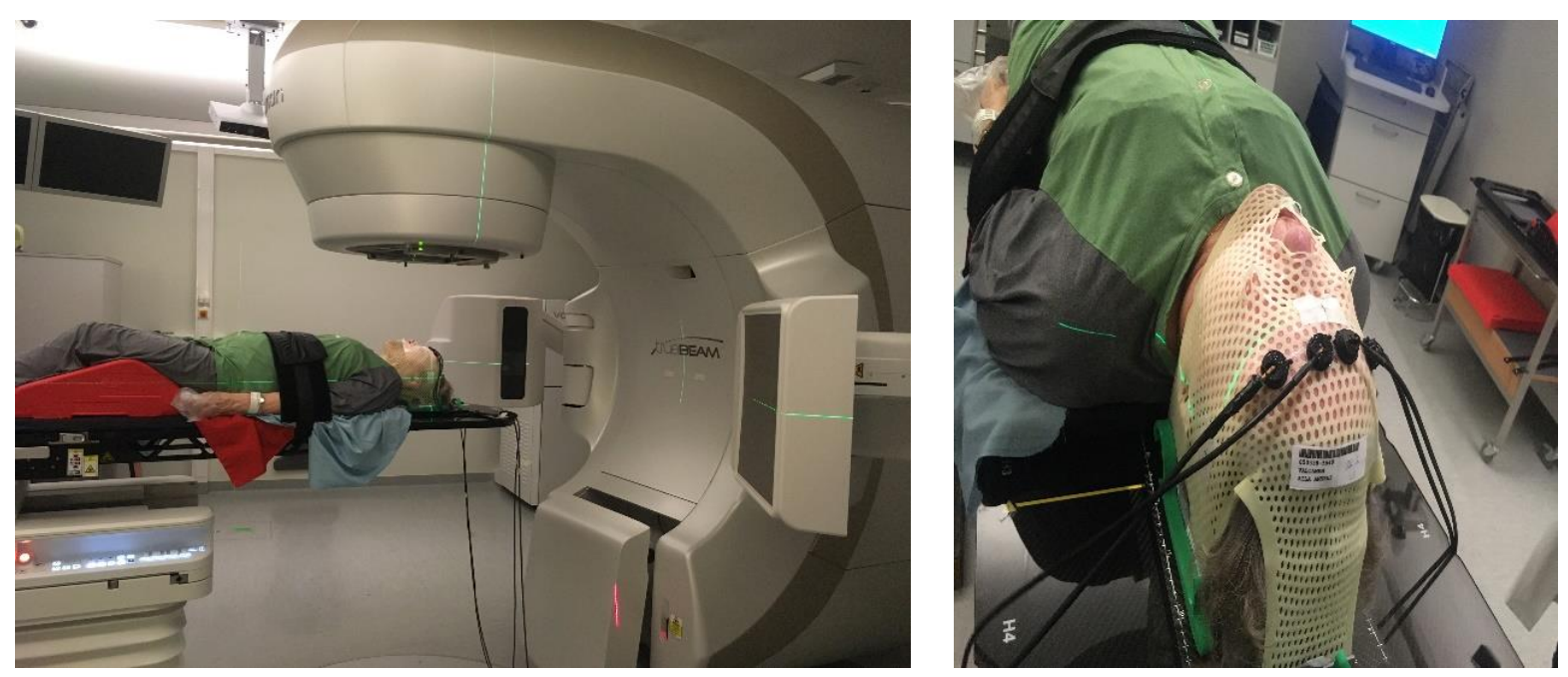

Figure 1. On the left, patient positioned in the treatment position in the medical linear accelerator (TrueBeam, Varian Medical Systems). On the right optical fibres perpendicular to the skin are attached on the mask at source-detector distance of $3 \mathrm{~cm}$. In the most types of external radiotherapy, like brain, head and neck area, patients have to wear a mask during the treatment to ensure the correct patient positioning to make the treatment spatially accurate and to avoid patient movement. Plastic mesh mask is the common mask used in brain radiotherapy, which is thermoplastic material and can be molded to perfectly fit the patient's head shape.

Ionizing radiation inside the radiotherapy treatment room poses high demands on the materials and devices used in there. In the case of a NIRS device, all optical fibres and optodes must be free of ferromagnetic metals, since these will be attached on the subject's head in the area of ionizing radiation. However, the NIRS device itself can be placed at a distance from the accelerator or outside radiotherapy room using optical fibres. In our setup, we placed only optical fibres and optodes in the treatment room and interfaced the units in the control room by a cable entry. Optical fibres used in the NIRS device are 10 meters long having 4 light source inputs and one output tip to enable mixing of four narrow band wavelengths, each modulated at a specific frequency [8]. Light sources are high-power light emitting diodes having wavelengths of 690 $\mathrm{nm}, 810 \mathrm{~nm}, 830 \mathrm{~nm}$ and $980 \mathrm{~nm}$ [9]. The fibre output tip with a 90 degree bend is attached on forehead using a 3D printed fibre clips which were clued on the mask commonly used in radiotherapy, see Figure 1 (on the right). The diameter of the fibre tip is $2.5 \mathrm{~mm}$ and source-detector distance is $3 \mathrm{~cm}$. All fibres have the same physical dimensions.

Measured raw NIRS time courses were converted into two time courses representing temporal changes in $\mathrm{HbO}, \mathrm{Hb}$ and HbT concentrations using Matlab-based NIRS data analysis tool HoMer2. HoMer2 is open source software which calculates concentration changes of $\mathrm{Hb}, \mathrm{HbO}$ and $\mathrm{HbT}$ automatically, based on the Modified Beer-Lambert law (MBLL) [10]. The basics of the program can be found in the publication by Huppert et al.[11]. In the performed analysis, wavelengths of $690 \mathrm{~nm}$ and $830 \mathrm{~nm}$ were used.

\subsection{Radiotherapy procedure}

Patient ( 82 years old) subjected to WBRT was monitored. Patient had secondary malignant neoplasm of brain due the lung cancer. WBRT treatment for $20 \mathrm{~Gy}$ in $4.0 \mathrm{~Gy}$ fractions was individually planned for the patient. Delivery of radiation dose was implemented using FIMRT technique. Treatment plan consisted of left side (6 MV, $213.5 \mathrm{MU}$, collimator angle $327^{\circ}$, gantry angle $\left.90^{\circ}\right)$ and right side $\left(6 \mathrm{MV}, 220.1 \mathrm{MU}\right.$, collimator angle $33^{\circ}$, gantry angle $270^{\circ}$ ) radiation fields (field size: $20.5 \mathrm{~cm} \times 14.9 \mathrm{~cm}$ ) shaped with multileaf collimator (MLC). Both main fields included one $6 \mathrm{MV}$ subfield $(9.2 \mathrm{MU}$ and 15.0 MU respectively). The implemented FIMRT technique resulted to the whole brain dose coverage of 95-107\% from prescribed dose. In all fields the dose rate was $600 \mathrm{MU} / \mathrm{min}$. 


\section{RESULTS}

Figure 2 shows full band responses of $\mathrm{HbO}$ and $\mathrm{HbR}$ for main field irradiation monitored from forehead in the treated area. During the $22 \mathrm{~s}$ irradiation (starts at $\mathrm{t}=10$ ), $\mathrm{HbO}$ shows immediate increase in signal amplitude and, on the right, $\mathrm{HbR}$ shows an antisymmetric decrease.
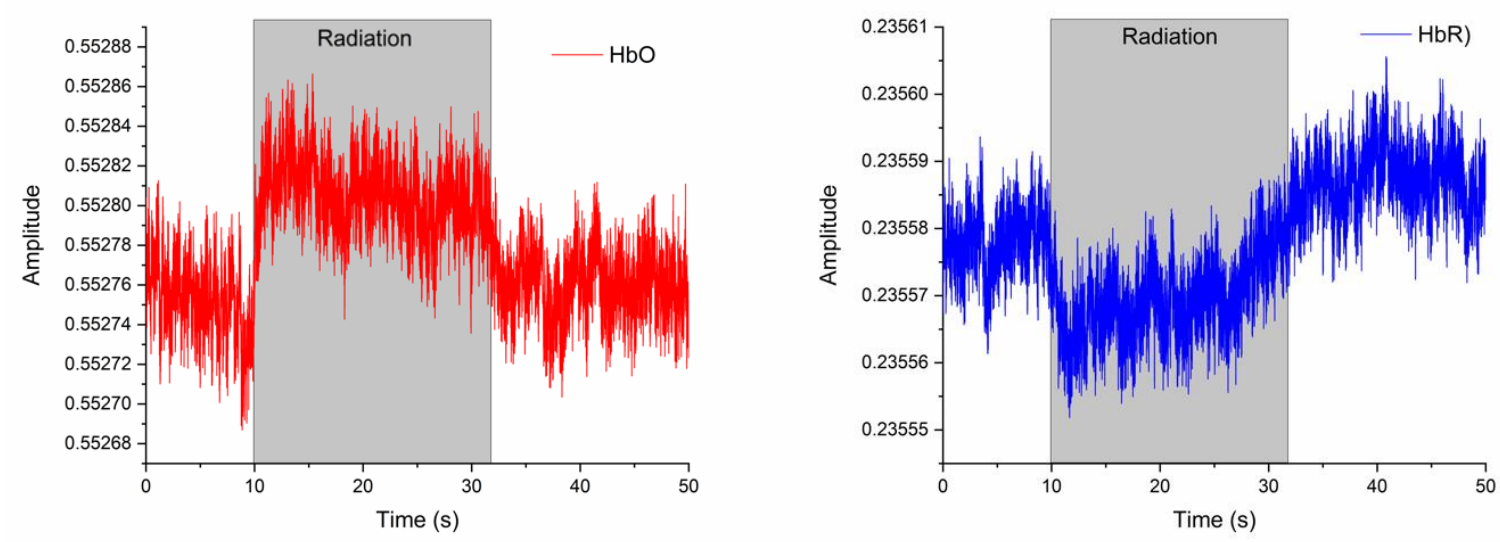

Figure 2. Full-band average responses $(\mathrm{n}=4)$ of $\mathrm{HbO}$ and $\mathrm{HbR}$ during radiation, colored with grey area.

Figure 3 shows averaged responses and its variance $(n=4)$ of $\mathrm{HbO}$ and $\mathrm{HbR}$ for irradiation. In all 4 measurements, during irradiation, a similar transient increase following with a slight decrease in signal strength of $\mathrm{HbO}$ can be observed while HbR has an antisymmetric notch.
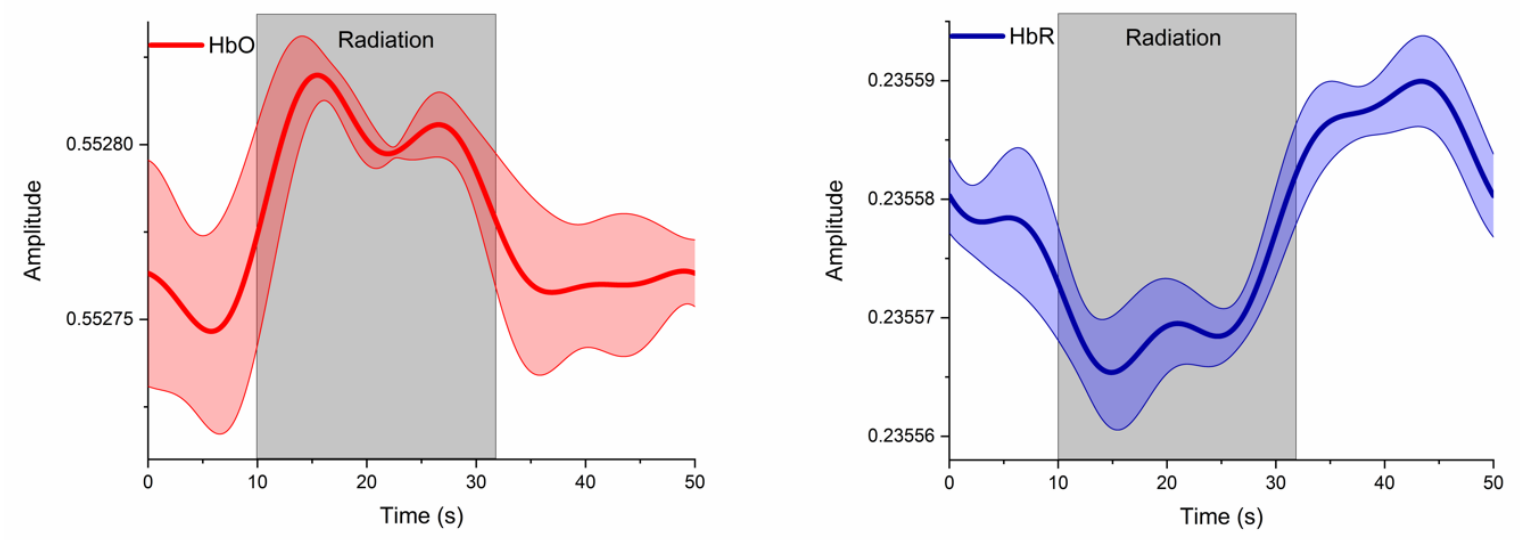

Figure 3. Band-pass filtered $(0.009-0.08 \mathrm{~Hz})$ averaged responses with variance $(\mathrm{n}=4)$ of $\mathrm{HbO}$ and $\mathrm{HbR}$ for irradiation.

\section{DISCUSSION}

Our results display instant cerebral haemodynamic response recorded by non-invasive fNIRS during clinical radiotherapy. Furthermore, this study demonstrate that it is feasible to monitor the immediate radiation induced changes of $\mathrm{HbO}$ and $\mathrm{HbR}$ in the human brain in vivo.

Previous studies monitoring tissue oxygenation, $\mathrm{HbT}$ and blood flow in the course of radiotherapy, suggest increased blood flow, tissue oxygenation and thus higher oxygen supply to the tumor in the first few weeks of the therapy [4],[5],[13]. These longitudinal responses are in line with tumor and tissue responses mechanisms in radiotherapy, i.e. reoxygenation of the tumor cells. The tumor responses and tumor models indicate that the reoxygenation occurs due tumor shrinkage, decreased oxygen consumption, and increased perfusion [14]. However, our results showing instant responses in $\mathrm{HbO}$ and 
$\mathrm{HbR}$ during the irradiation followed by disappearance of the effect after the radiation ceases, suggest to the more immediate processes which would have been unobservable in these previous studies as the measurements have been conducted between irradiations.

Increase in $\mathrm{HbO}$ during irradiation could be explained by reduction process of methaemoglobin (metHb). The superoxide radical ion $(\mathrm{O} 2 \bullet-)$, one of the reactive oxygen species (ROS) produced by ionizing radiation, can reduce haem groups in met $\mathrm{Hb}$ resulting in $\mathrm{HbO}$. Furthermore, the $\mathrm{O} 2 \bullet-$ can oxidize haem groups of $\mathrm{HbO}$ resulting in production of met $\mathrm{Hb}$, and by this process is replaced by the Superoxide dismutase process when the concentration of O2 - is sufficiently high [12]. Furthermore, the reduction of $\mathrm{HbR}$ could be related to the formation of aquo metHb [15].

Earlier studies, for instance, by Oshtrakh et al. show that X-ray irradiation leads to changes in haemoglobin oxygenation and protein destruction [16]. Polakovs et al. used Raman and FT-IR spectroscopy to observe radiation influence on blood of patients examined by radio-isotopes diagnosis (Tc99m). They suggest that radiation of blood leads to transition from haemoglobin to metHb with delocalization of iron from porphyrin plane [17]. Furthermore, a more recent study by Polakovs et al. examined metHb in human blood after radiation by linear accelerator. Particularly ionizing radiation change valence of iron from haemoglobin $(\mathrm{Fe} 2+)$ to metHb $(\mathrm{Fe} 3+)$ showed similar effects with various types of ionizing radiation [18].

However, further research is required because previous studies have been conducted in vitro, and after irradiation, suggesting to more permanent alterations in haemoglobin structure. Furthermore, it remains to be elucidated whether production of reactive oxygen species (ROS) (and thus, production of metHb and aquo metHb) is sufficiently high in our measurement setup to result in the measured temporal alterations in $\mathrm{HbO}$ and $\mathrm{HbR}$ levels.

\section{CONCLUSION}

To our knowledge, this is the first time the cerebral haemodynamics in clinical WBRT have been monitored during the irradiation. Our study demonstrates, that the online monitoring of temporal alterations in cerebral haemodynamics during irradiation is feasible. Evidently, irradiation causes noticeable immediate changes in cerebral haemodynamics. However, further studies must be conducted in order to better understand the interaction between the irradiation and its biomolecular responses, in vivo. Monitoring the real-time interactions between the subjected radiation dose and the haemodynamics would provide important tool for the researchers and clinicians in the field of radiotherapy.

\section{ACKNOWLEDGEMENTS}

This work was supported by Academy of Finland (grant 318347) and EDUFI Fellowships (P.K.).

\section{REFERENCES}

[1] Obrig, H., "NIRS in clinical neurology—a 'promising’tool?" Neuroimage 85, 535-546 (2014).

[2] Yu, B., "Quantitative optical spectroscopy and imaging for cancer diagnosis and treatment monitoring," 2016 Progress in Electromagnetic Research Symposium (PIERS), 2322-2322 (2016).

[3] Howe, F. A., Connelly, J. P., Robinson, S. P., Springett, R. and Griffiths, J. R., "The effects of tumour blood flow and oxygenation modifiers on subcutaneous tumours as determined by NIRS,", 75-81 (2005).

[4] Sunar, U., Quon, H., Durduran, T., Zhang, J., Du, J., Zhou, C., Yu, G., Choe, R., Kilger, A. and Lustig, R. A. , "Noninvasive diffuse optical measurement of blood flow and blood oxygenation for monitoring radiation therapy in patients with head and neck tumors: a pilot study," J.Biomed.Opt. 11(6), 064021 (2006).

[5] Dong, L., Kudrimoti, M., Irwin, D., Chen, L., Kumar, S., Shang, Y., Huang, C., Johnson, E. L., Stevens, S. D. and Shelton, B. J. , "Diffuse optical measurements of head and neck tumor haemodynamics for early prediction of chemoradiation therapy outcomes," J.Biomed.Opt. 21(8), 085004 (2016).

[6] Nyström, J., Geladi, P., Lindholm-Sethson, B., Rattfelt, J., Svensk, A. and Franzen, L., "Objective measurements of radiotherapy-induced erythema," Skin Research and Technology 10(4), 242-250 (2004).

[7] Mohammadkarim, A., Mokhtari-Dizaji, M., Kazemian, A. and Saberi, H., "Hemodynamic analysis of radiationinduced damage in common carotid arteries by using color Doppler ultrasonography," Ultrasonography 37(1), 43-49 (2018).

[8] Myllylä, T., Harju, M., Korhonen, V., Bykov, A., Kiviniemi, V. and Meglinski, I., "Assessment of the dynamics of human glymphatic system by near-infrared spectroscopy," Journal of biophotonics 11(8), e201700123 (2018). 
[9] Myllylä, T., Korhonen, V., Suraźyński, Ł., Zienkiewicz, A., Sorvoja, H. and Myllylä, R., "Measurement of cerebral blood flow and metabolism using high power light-emitting diodes," Measurement 58, 387-393 (2014).

[10] Cope, M. and Delpy, D. T., "System for long-term measurement of cerebral blood and tissue oxygenation on newborn infants by near infra-red transillumination," Medical and Biological Engineering and Computing 26(3), 289-294 (1988).

[11] Huppert, T. J., Diamond, S. G., Franceschini, M. A. and Boas, D. A., "HomER: a review of time-series analysis methods for near-infrared spectroscopy of the brain," Appl.Opt. 48(10), D280-D298 (2009).

[12] Sutton, H. C., Roberts, P. B., \& Winterbourn, C. C. (1976). The rate of reaction of superoxide radical ion with oxyhaemoglobin and methaemoglobin. The Biochemical journal, 155(3), 503-510.

[13] Mayr, N. A., Yuh, W. T., Magnotta, V. A., Ehrhardt, J. C., Wheeler, J. A., Sorosky, J. I., Davis, C. S., Wen, B., Martin, D. D. and Pelsang, R. E. , "Tumor perfusion studies using fast magnetic resonance imaging technique in advanced cervical cancer: a new noninvasive predictive assay," International Journal of Radiation Oncology* Biology* Physics 36(3), 623-633 (1996).

[14] Wachsberger, P., Burd, R. and Dicker, A. P., "Tumor response to ionizing radiation combined with antiangiogenesis or vascular targeting agents: exploring mechanisms of interaction," Clin.Cancer Res. 9(6), 19571971 (2003).

[15] Oshtrakh, M., "Comparison of the oxyhemoglobin deoxygenation and radiolysis by $\hat{I}^{3}$-rays and electrons: Study by MÃđIssbauer spectroscopy," Nuclear Instruments and Methods in Physics Research Section B: Beam Interactions with Materials and Atoms 185(1-4), 129-135 (2001).

[16] Oshtrakh, M., Semionkin, V., Kopelyan, E. and Milder, O., "Mössbauer and positron life-time study of oxyhemoglobin solution irradiated by electrons," Radiat.Phys.Chem. 55(5-6), 549-554 (1999).

[17] Polakovs, M., Mironova-Ulmane, N., Kurjane, N., Reinholds, E. and Grube, M., "Micro-Raman scattering and infrared spectra of hemoglobin," Sixth International Conference on Advanced Optical Materials and Devices (AOMD-6), 714214 (2008).

[18] Polakovs, M., Mironova-Ulmane, N., Pavlenko, A. and Aboltins, A., "Determination of methemoglobin in human blood after ionising radiation by EPR," IOP Conference Series: Materials Science and Engineering, 012028 (2015). 